\title{
Managing the Nutritional Requirements of Vegetarian and Vegan Mothers During Pregnancy
}

\author{
Amanda Strombom* \\ Stewart Rose, Plant-Based Diets in Medicine, USA
}

Submission: January 31, 2020; Published: February 06, 2020

*Corresponding author: Amanda Strombom, Stewart Rose, Plant-Based Diets in Medicine, USA

\begin{abstract}
Vegan pregnant women have a lower-than-average rate of cesarean delivery, less postpartum depression, and lower neonatal and maternal mortality. Well-planned plant-based diets have been confirmed as safe during pregnancy and lactation, as well as conferring additional health advantages. However, vegan diets that are highly restricted in calories and nutrients may give birth to infants whose weights are significantly lower than expected, so it's important for physicians to know how to manage such pregnancies. Vegetarians and vegans have significantly lower BMIs, on average, and a lower risk of hypothyroidism, which are the most significant risk factors for Gestational Diabetes Mellitus. They also have a lower risk of pre-eclampsia and gallstone disease. Comorbidities such as chronic kidney disease and hypercholesterolemia can be effectively treated with a plant-based diet. Fear that the risk of the birth defect hypospadias was increased by following a vegetarian diet have proved unwarranted.

Protein intake of vegan women should be increased by $10 \%$ while pregnant, and they should be encouraged to consume a wide variety of plant-based foods. Adequate sources of Omega 3 fats, calcium, iodine, vitamins B12 and D must be ensured, and a multi-micronutrient supplement tablet containing the recommended daily allowance (RDA) of several vitamins and minerals is recommended. A plant-based diet offers significant advantages in reducing complications of pregnancy as well as treating comorbidities such as Type II diabetes. While attention to vitamin B12 and other nutrients, when indicated, is important, a vegan pregnancy is not very different from the more common pregnancies.

Keywords: Birth defects; Birthweight; Gestational diabetes; Lactation; Plant-based; Pre-eclampsia; Pregnancy; Pregnant; Vegan; Vegetarian
\end{abstract}

\section{Introduction}

Although often framed in terms of lacking, vegan diets are actually rich in a wide variety of foods: grains, legumes (including soy and its derivatives), vegetables, fruits, nuts and seeds, vegetable fats, and herbs and spices [1,2]. The American Academy of Nutrition and Dietetics position statement on plantbased diets confirms their safety during pregnancy and lactation and that they may confer additional health advantages [1] Vegan pregnant women have a lower-than-average rate of cesarean delivery, less postpartum depression, and lower neonatal and maternal mortality, with no complications or negative outcomes that are higher than average [3-5]. Both maternal malnutrition and overnutrition are associated with subsequent diabetes in the offspring. Pregnancy represents a window of opportunity for health care providers to change dietary patterns toward habits that will be healthier for the individual now, as well as impacting the mother and child in the future [6]. A growing number of people are choosing to be vegetarians and vegans in the United States.
According to a survey conducted by Vegetarian Times there are about 7.3 million vegetarians in United States. Of those about 1 million are vegan [7]. These numbers are likely to rise especially with the increased availability of meat substitutes. It's important for the physician to know how to manage their pregnancies.

\section{Birthweight concerns for vegetarian mothers}

Several studies have looked at the birthweight of infants born to vegetarian mothers. Five studies showed a lower birthweight in the children of vegetarian mothers [8-12]. However the result was significant in only one study. Conversely, birthweight and length were higher in children of vegetarian mothers in two studies. [13, 14].

Results of all these studies are affected by country, ethnicity, socioeconomic status, and other health related behaviors such as smoking and access to prenatal care $[15,16]$. This is a crucial issue also reflected in the different social patterns in rich, western 
countries, in which vegan-vegetarian diets are often chosen in the quest for a healthier lifestyle, compared with low income countries, in which the nutritional deficits may be linked to forced limitations in the availability of food [17].

One study found that significantly higher birthweight (mean 99g) above that of non-vegetarians, and involved a community of Seventh-Day Adventists [14]. This is notable because, as a group, Seventh-day Adventists follow a healthy lifestyle. The difference in this study was therefore most likely related to diet.

Another study showed that the birthweight of children of vegan mothers is lower than that of the omnivorous mothers' children, but the values of this anthropometric parameter were all within the accepted range. No significant difference has emerged from the comparison for the length, cranial circumference and BMI at birth between the vegan group and the omnivorous group [18].

Vegan diets that are highly restricted in calories and nutrients, in contrast to well-planned vegan diets, give birth to infants whose weights are significantly lower than expected [12]. Concerns about vegan diets during pregnancy, breastfeeding, infancy, and childhood arose in the past $[17,19,20]$, but this was due to the fact that although being categorized as "vegan", the investigated subjects were following highly restrictive diets, not respecting all the criteria required to define the diet as being well-planned.

\section{Risk of gestational diabetes}

Observational studies show consistent evidence that increased BMI and hypothyroidism are the strongest risk factors for Gestational Diabetes Mellitus (GDM) [21]. Vegetarians and vegans have significantly lower BMIs on average. A study of American vegetarians and vegans found that that vegetarians had a mean BMI of 25.7 and vegans a mean BMI of 23.6 [22]. A European study found the average BMI of vegetarians and vegans to be 23.3 and 22.4 respectively for men and 22.8 and 21.8 for women [23] A study of German vegans found an average BMI of 22.3 [24]. While vegan diets are associated with lower body weight, which may protect against hypothyroidism, a lower risk of hypothyroidism among vegans exists even after controlling for BMI and potential demographic confounders. One study showed that following a vegan diet tended to be associated with a $22 \%$ reduced risk of hypothyroidism, although statistical significance was not quite attained [25].

A high intake of fiber during pregnancy also seems to be particularly beneficial in preventing GDM. Indeed, maternal diets characterized by low intakes of fiber and a high glycemic load seem to be associated with an increased risk of GDM, In particular, an increment of $10 \mathrm{~g} /$ day in total fiber intake was found to be associated with a $26 \%$ risk reduction in GDM and an increment of $5 \mathrm{~g} /$ day in cereal or fruit fiber intake was associated with a $23 \%$ or $26 \%$ reduction in GDM, respectively [26]. The vegetarian diet was also associated with a lower incidence of excessive gestational weight gain [27].
Higher intake of animal protein, in particular red meat, was significantly associated with a greater risk of GDM. Several major food sources of animal protein, such as red meat, were positively associated with the risk of GDM [28]. In particular, a meat-centered diet was associated with significantly higher GDM risk in parous and obese women [29] By contrast, higher intake of vegetable protein, specifically nuts, was associated with a significantly lower risk. Substitution of vegetable protein for animal protein was associated with a lower risk of GDM [30].

\section{Risk of gallstones}

The incidence rates of biliary sludge (a precursor to gallstones) and gallstones are up to $30 \%$ and $12 \%$, respectively, during pregnancy and postpartum, and gallbladder disease is the most common non obstetrical cause of maternal hospitalization in the first year postpartum [31,32]. Prevention of gallstone disease is especially important during pregnancy, as cholecystectomy is problematic and medication can carry risks. In pregnancy, the incidence of cholesterol gallstones is increased by strong risk factors including obesity, serum leptin and extreme hypercholesterolemia. A plant-based diet has been shown to lower the risk of obesity, reduce leptin and eliminate cholesterol from the diet, thus reducing serum cholesterol and therefore gallstone risk during pregnancy without the risk that drug treatment may entail. [33].

\section{Risk of Pre-eclampsia}

Vegans may have a lower risk of preeclampsia. The risk of preeclampsia in the general population is about $3 \%$. However, in a study of 775 vegan pregnancies, only one case of preeclampsia was noted, [4] giving a rate of only about $01 \%$. Other less specific studies have shown decreased risk with higher consumptions of fruits and vegetables. In a Norwegian study, women with high scores on a dietary pattern characterized by vegetables, plant foods, and vegetable oils, were at $28 \%$ decreased risk of preeclampsia for the highest tertile vs. the lowest tertile [34].

This could be related to the theory that the risk of preeclampsia is directly associated with a high consumption of fat and sugar and a low intake of fiber, since plant-based dietary patterns generally provide low amounts of fat and sugar and higher quantities of fiber [35]. One study showed that the highest quartile of soluble fiber intake reduced the risk of preeclampsia by $70 \%$ and insoluble fiber by $65 \%$ compared to the lowest quartile [36].

\section{Risk of birth defects}

Early concerns were expressed about the birth defect hypospadias (a congenital malformation in which the opening of the penile urethra occurs on the ventral side of the penis) in association with vegetarian diets. However, this has turned out to be unwarranted according to a large study [37]. Other concerns raised over the use of soy phytoestrogens also seem to be unwarranted. According to one study, a higher intake of some phytoestrogens was associated with reduced risks of delivering 
infants with hypospadias, even after adjustment for several covariates. This finding applied to overall intake of phytoestrogens as well as intake of specific phytoestrogens, so phytoestrogens may actually be protective of hypospadias [38].

Pregnant women following a well-planned plant-based diet, being naturally higher in folate, may have a reduced risk of an infant being born with spina bifida or anencephaly [39]. In contrast, a western diet (including high intake of meat) was shown to increase by around 2-fold the risk of offspring with orofacial cleft palate [40].

\section{Pregnant with chronic kidney disease}

Using the most recent classification system, 3\% of women of childbearing age are affected by Chronic Kidney Disease (CKD) $[41,42]$ For these women, the risk of an adverse pregnancy rises very significantly, even in stage 1 [43]. Despite vast improvements in fetal outcomes, pregnancy in women with CKD is fraught with hazards; worsening renal function and complications such as preeclampsia and premature delivery are common [44].

During the pregnancy of a patient with CKD, the amount of protein in the dietmust be balanced between the goal of diminishing hyperfiltration and increasing metabolic needs of pregnancy [45]. Due to the fact that pregnancy induces hyperfiltration, diets with restricted amount of protein should be beneficial in this group of patients $[46,47]$. Vegan or vegetarian supplemented low-protein diets in pregnant women with stages 3-5 CKD reduce the risk of small-for-gestational-age babies, without detrimental effects on kidney function or proteinuria in the mother [48].

CKD during pregnancy presents a clinical challenge, especially considering the paucity of therapeutic tools available in pregnant women. One study investigated the feasibility of supplemented vegetarian low-protein diets in pregnancy, as a "rescue treatment" for severe CKD and or proteinuria [46]. None of the 11 patients needed renal replacement therapy within the 6 months before delivery. No patient complained of side effects, nor developed hyperkalemia or hypercalcaemia. All babies were well at 1 month post delivery, and 7.5 years later [46]. A supplemented vegetarian low-protein diet (0.6-0.7g/kg per day) turned out to be sufficient for the maintenance of satisfactory nutritional status during the pregnancy and after delivery, even in breast-feeding women [49]. For pregnant women with focal segmental glomerulosclerosis, a study showed that a moderately protein restricted, keto analogue supplemented, plant-based diet helped control proteinuria [50]. Another study reviewed the results obtained over 15 years of treating pregnant women with CKD on moderately restricted plant-based low-protein diets. It confirms that such a diet is a safe option in the management of pregnant CKD patients [51]. A trend towards better preserved fetal growth was observed. These results indicate that the treatment of pregnant CKD women on moderately restricted plant-based low-protein diet is a safe option in the management of pregnant CKD.

\section{Clinical considerations during pregnancy and lactation on a vegan diet}

Appropriately planned vegetarian, including vegan, diets are healthful, nutritionally adequate, and may provide health benefits for the prevention and treatment of certain diseases. These diets are appropriate for all stages of the life cycle, including during pregnancy and lactation [1]. Protein intakes should be increased by $10 \%$ in vegan pregnant and lactating women, compared with non-pregnant adult vegetarians. [2,52,53]. Additional servings of grains, protein-rich plant foods (legumes, soy milk, soy yogurt, tofu, tempeh, and meat analogs based on wheat or soy protein) and nuts and seeds should be consumed by vegan women during the second and third trimester of pregnancy and during breastfeeding to meet these increased protein requirements [53].

The patient should be advised to consume large amounts and a wide variety of plant foods, emphasizing the intake of whole or minimally processed foods: a vegan diet can be nutritionally adequate when meeting the calorie requirements from a variety of nutrient-dense foods, mainly unprocessed, belonging to all the plant food groups. It is recommended that the amount of vegetable fats be limited, as suggested by the Dietary Reference Intakes (DRIs), in order to limit excess calories and not displace more nutrient-dense foods. However, DHA plays an important role in pregnancy and lactation. Infants of vegetarian mothers appear to have lower cord and plasma DHA than do infants of nonvegetarians although the functional significance of this is not known $[9,13]$. Vegetable fats should therefore be chosen carefully, in order to consume good sources of omega-3 fatty acids such as walnuts, ground chia seeds and flax seeds (which can be converted to DHA) and monounsaturated oils, while avoiding trans fats and tropical oils (coconut, palm, and palm kernel oils), to emphasize the efficiency of the omega-3 metabolic pathway. DHA supplements made from microalgae are available and can be a supplement for patients when indicated. Note that during infancy and early childhood, fats should not be limited but should still be carefully chosen $[2,53,54]$.

Adequate amounts of calcium should be consumed, and vitamin D status should be checked. Additional calcium can be obtained by increasing the intakes of calcium-rich foods from plant sources. Conversely, since a plant-based diet cannot provide adequate amounts of vitamin $\mathrm{D}$, the recommendations for vitamin $\mathrm{D}$ are the same as for the general population $[2,53,54]$. Adequate amounts of vitamin B12 are essential, as deficiency increases the risk of neural tube defects in the infant [55]. The intake of a reliable source of vitamin B12 is fundamental to a well-planned vegetarian diet, as vitamin B12 status can be compromised, over time, in all vegetarian subjects who do not supplement it. $[2,53,54]$ Since B12 deficiency can occur during pregnancy regardless of the type of diet, because of store depletion due to higher demands [56], the use of a specific vitamin B12 supplement represents the most reliable way or ensuring an adequate B12 status during vegan pregnancy 
[57]. Increased caloric and nutrient intakes are recommended to meet the demands of the rapidly growing fetus and the increased physiological requirements of the mother, especially for folate, iron, iodine, and copper [58,59]. Although nutrient intakes should preferably come from a variety of food sources, it is possible that pregnant women and those of childbearing age won't meet their needs for some nutrients through diet alone [60,61]. As such, prenatal dietary supplements are generally recommended during pregnancy $[62,63]$. and were used by about $75 \%$ of pregnant women in a nationally representative US sample [60].

However, prenatal dietary supplements provide variable nutrient content and the number of nutrients included in dietary supplement formulations is not standard [62]. Concerns exist of excessive intakes of some nutrients during pregnancy, especially folic acid and vitamin A, $[64,65]$ while low intake of iron and iodine in reproductive-aged US women has also been of concern [66]. Thus, ensuring that pregnant and reproductive-aged women have adequate, but not excessive, dietary intakes is crucial during this critical life stage [67].

A multimicronutrient supplement tablet containing the recommended daily allowance (RDA) for pregnancy of several vitamins and minerals: vitamin $A$, vitamin $B 1$, vitamin $B 2$, niacin, vitamin B6, vitamin B12, folic acid, vitamin $C$, vitamin $D$, vitamin $E$, iron, copper, selenium, and zinc, should be prescribed [68].

The patient should be questioned about their sources of iodine, such as iodized salt and seaweed. If these are lacking in their diet then a prenatal supplement containing iodine is important. One advantage of a plant-based diet is that it can prevent and treat comorbidities. When comorbidities are present, it is important to titrate any medications when initiating a plant-based diet as the effects of the plant-based diet becomes evident.

\section{Ensuring good quality breast milk}

The breast milk of vegetarian women is similar in composition to that of nonvegetarians and is nutritionally adequate. Commercial infant formulas should be used if infants are not breastfed or are weaned before 1 year of age. Soy formula is the only option for non-breastfed vegan infants. Other preparations including regular soymilk, rice milk, and homemade formulas should not be used to replace breast milk or commercial infant formula [69]. The period of lactation is extremely important for growing patterns of infants and the effectiveness of breastfeeding depends on maternal nutritional status. A lack of macro- and micronutrient intake during lactation may lead to the reduction of micronutrients and energy content in breast milk that could potentially lead to severe illness in the breastfed infant [70].

Breast milk of vegan women following well-planned vegan diets including a reliable source of vitamin B12 provides adequate nutrition for their breastfed infants [53]. According to one study, $20 \%$ of study participants were classified as having low breastmilk vitamin B-12 concentrations $(<310 \mathrm{pmol} / \mathrm{L})$, independent of maternal diet pattern [57]. In this study, $85 \%$ of participants categorized as having low vitamin B-12 were taking vitamin B-12 supplements at doses in excess of the RDA, which suggests that more research is needed to determine breast-milk adequacy values [57] Milk from breastfeeding vegan mothers provides adequate vitamin B12 in infants only if vegan mothers are supplementing with B12 sufficiently larger than the recommended amounts [57]. Given the safety of vitamin B12 supplements, [71] large amounts should ensure sufficiency.

Although containing $100 \%$ of the RDA for vitamin B12, common pre- and postnatal multivitamins have been negatively associated with low B12 concentration in breastmilk of vegan women, because only a fraction of the B12 they provide is absorbed $[2,56]$. Pregnant and lactating vegan mothers should be encouraged to take an individual B12, not multivitamin, supplement and to dissolve it under the tongue or chew it slowly in order to increase absorption [2,57].

Breast milk DHA is lower in vegans and lacto-ovovegetarians than in nonvegetarians [72,73] Because of DHA's beneficial effects on gestational length, infant visual function, and neurodevelopment, pregnant and lactating vegetarians and vegans should choose the DHA precursor linolenic acid in their diet (ground flaxseed, ground chia seeds, walnuts, canola oil, soybean oil), food sources of DHA (foods fortified with DHA-rich microalgae) or use a microalgae-derived DHA supplement $[74,75]$. Supplementation with ALA, a DHA precursor, in pregnancy and lactation may not be sufficient to increase infant DHA levels or breast milk DHA concentration [76,77].

\section{Discussion}

A plant-based diet offers significant advantages in reducing complications of pregnancy. It also provides a safe and effective treatment for comorbidities that are happening earlier in life such as Type II diabetes, extreme hypercholesterolemia, and auto-immune diseases such as hypo and hyperthyroidism, plus it helps prevent gallstones to which pregnant women are more susceptible. The American Academy of Nutrition and Dietetics position statement on plant-based diets confirms their safety during pregnancy and lactation, and that they may confer additional health advantages.

Supplementation with vitamin B12 is essential, since deficiency increases the risk of neural tube defects. Some vegan women resist vitamin B12 supplementation out of philosophical objections. They seem to want to show that a plant-based diet is sufficient by itself, and they misunderstand that since vitamin B12 is synthesized by soil bacteria, it is our modern hygiene practices (scrupulously washing our vegetables) that results in less Vitamin B12 being absorbed by those not consuming animal products. It is likely that if more women following a plant-based diet, as a group, ensured adequate vitamin B12 and iodine intake, their health advantages in pregnancy would be even greater than they already 
are. However, while attention to vitamin B12 and other nutrients is important, a vegan pregnancy is not very different from the more common pregnancies, while conferring considerable benefits.

\section{References}

1. Melina V, Craig W, Levin S (2016) Position of the Academy of Nutrition and Dietetics: Vegetarian Diets. J Acad Nutr Diet 116(12): 1970-1980.

2. Agnoli C, Baroni L, Bertini I, Ciappellano S, Fabbri A, et al. (2017) Position paper on vegetarian diets from the working group of the Italian Society of Human Nutrition. Nutr Metab Cardiovasc Dis 27(12): 1037-1052.

3. Gaskin IM (2002) Spiritual Midwifery. Book Publishing Company, Summertown (TN), USA.

4. Carter J, Furman T, Hutcheson H (1987) Preeclampsia and reproductive performance in a community of vegans. South Med J 80(6): 692-697.

5. Aronson D (2007) Advice for Vegan Mothers-to-Be - Nine Months of Proper Nutrition. Today's Dietitian 12: 38.

6. Jovanovic L (2004) Nutrition and pregnancy: the link between dietary intake and diabetes. Curr Diab Rep 4(4): 266-272.

7. Vegetarian Times Editors (2008) Vegetarianism in America.

8. Wen X, Justicia-Linde F, Kong K, Zhang C, Chen W, et al. (2013) Associations of diet and physical activity with the three components of gestational weight gain. Am J Epidemiol 11(Suppl): S1-S181.

9. Reddy S, Sanders TAB, Obeid O (1994) The influence of maternal vegetarian diet on essential fatty acid status of the newborn. Eur J Clin Nutr 48(5): 358-368.

10. Ward RJ, Abraham R, McFadyen IR, Haines AD, North WR, et al. (1988) Assessment of trace metal intake and status in a Gujerati pregnant Asian population and their influence on the outcome of pregnancy. $\mathrm{Br}$ ] Obstet Gynaecol 95(7): 676-682.

11. Campbell-Brown M, Ward RJ, Haines AP, North WR, McFadyen IR, et al. (1985) Zinc and copper in Asian pregnancies--is there evidence for a nutritional deficiency? Br J Obstet Gynaecol 92(9): 875-885.

12. Thomas J, Ellis F (1977) The health of vegans during pregnancy. Proc Nutr Soc 36(1): 46A.

13. Lakin V, Haggarty P, Abramovich D, Ashton J, Moffat C, et al. (1998) Dietary intake and tissue concentration of fatty acids in omnivore vegetarian and diabetic pregnancy. Prostaglandins Leukot Essent Fatty Acids 59(3): 209-220.

14. Fønnebø V (1994) The healthy Seventh-Day Adventist lifestyle: what is the Norwegian experience? Am J Clin Nutr 59(5 Suppl): 1124S-1129S.

15. Tofail F, Persson LÅ, Arifeen SE, Hamadani JD, Mehrin F, et al. (2008) Effects of prenatal food and micronutrient supplementation on infant development: a randomized trial from the Maternal and Infant Nutrition Interventions, Matlab (MINIMat) study. Am J Clin Nutr 87(3): 704-711.

16. McDonald EC, Pollitt E, Mueller W, Hsueh AM, Sherwin R, et al. (1981) The Bacon Chow study: maternal nutrition supplementation and birth weight of offspring. Am J Clin Nutr 34(10): 2133-2144.

17. Piccoli G, Clari R, Vigotti F, Leone F, Attini R, et al. (2015) Veganvegetarian diets in pregnancy: danger or panacea? A systematic narrative review. BJOG 122(5): 623-633.

18. Ferrara P, Sandullo F, Ruscio FD, Franceschini G, Peronti B, et al. (2019) The impact of lacto-ovo-/lacto-vegetarian and vegan diets during pregnancy on the birth anthropometric parameters of the newborn. J Matern Fetal Neonatal Med, pp. 1-7.
19. Schürmann S, Kersting M, Alexy U (2017) Vegetarian diets in children: a systematic review. Eur J Nutr 56(5):1797-1817.

20. Richter M, Boeing H, Grünewald-Funk D, Heseker H, Kroke A, et al (2016) Vegan diet. Position of the German Nutrition Society (DGE) Ernahrungs Umschau 63: 92-102.

21. Giannakou K, Evangelou E, Yiallouros P, Christophi C, Middleton N, et al. (2019) Risk factors for gestational diabetes: An umbrella review of meta-analyses of observational studies. PLoS One 14(4): e0215372.

22. Tonstad S, Butler T, Yan R, Fraser G (2009) Type of Vegetarian Diet, Body Weight, and Prevalence of Type 2 Diabetes. Diabetes Care 32(5): 791-796.

23. Bradbury K, Crowe F, Appleby P, Schmidt J, Travis R, et.al. (2014) Serum concentrations of cholesterol, apolipoprotein A-I, and apolipoprotein B in a total of 1694 meat-eaters, fish-eaters, vegetarians, and vegans. Eur J Clin Nutr 68(2): 178-183.

24. Waldmann A, Koschizke J, Leitzmann C, Hahn A (2005) German vegan study: diet, life-style factors, and cardiovascular risk profile. Ann Nutr Metab 49(6): 366-372.

25. Tonstad S, Nathan E, Oda K, Fraser G (2013) Vegan diets and hypothyroidism. Nutrients 5(11): 4642-4652.

26. Zhang C, Liu S, Solomon CG, Hu FB (2006) Dietary fiber intake, dietary glycemic load, and the risk for gestational diabetes mellitus. Diabetes Care 29(10): 2223-2230.

27. Streuling I, Beyerlein A, Rosenfeld E, Schukat B, Kries Rv, et al. (2011) Weight gain and dietary intake during pregnancy in industrialized countries - a systematic review of observational studies. J Perinat Med 39(2): 123-129.

28. Zhang C, Schulze MB, Solomon CG, Hu FB (2006) A prospective study of dietary patterns, meat intake and the risk of gestational diabetes mellitus. Diabetologia 49(11): 2604-2613.

29. Schoenaker DAJM, Soedamah-Muthu SS, Callaway LK, Mishra GD (2015) Pre-pregnancy dietary patterns and risk of gestational diabetes mellitus: results from an Australian population-based prospective cohort study. Diabetologia 58(12): 2726-2735.

30. Bao W, Bowers K, Tobias DK, Hu FB, Zhang C, et al. (2013) Prepregnancy Dietary Protein Intake, Major Dietary Protein Sources, and the Risk of Gestational Diabetes Mellitus. Diabetes Care 36(7): 2001-2008.

31. Ko C (2006) Risk factors for gallstone-related hospitalization during pregnancy and the postpartum. Am J Gastroenterol 101(10): 22632268.

32. Lydon-Rochelle M, Holt VL, Martin DP, Easterling TR (2000) Association between method of delivery and maternal rehospitalization. JAMA 283(18): 2411-2416.

33. Rose S, Strombom A (2018) A comprehensive review of the prevention and treatment of heart disease with a plant-based diet. J Cardiol \& Cardiovas Ther 12(5): 555847.

34. Brantsæter AL, Haugen M, Samuelsen S, Torjusen H, Trogstad L, et al. (2009) A dietary pattern characterized by high intake of vegetables, fruits, and vegetable oils Is associated with reduced risk of preeclampsia in nulliparous pregnant Norwegian women. J Nutr 139(6): 1162-1168.

35. Frederick I, Williams M, Dashow E, Kestin M, Zhang C, et al (2005) Dietary fiber, potassium, magnesium and calcium in relation to the risk of preeclampsia. J Reprod Med 50(5): 332-344.

36. Qiu C, Coughlin KB, Frederick IO, Sorensen TK, Williams MA, et al. (2008) Dietary Fiber Intake in Early Pregnancy and Risk of Subsequent Preeclampsia. Am J Hypertens 21(8): 903-909. 
37. Carmichael S, Ma C, Feldkamp M, Munger R, Olney R, et al. (2012) Nutritional factors and hypospadias risks. Paediatr Perinat Epidemiol 26(4): 353-360.

38. Carmichael SL, Cogswell ME, Ma C, Gonzalez-Feliciano A, Olney R, et al. (2013) Hypospadias and Maternal Intake of Phytoestrogens. Am J Epidemiol 178(3): 434-440.

39. Centers for Disease Control and Prevention (CDC) (2004) Spina bifida and anencephaly before and after folic acid mandate--United States, 1995-1996 and 1999-2000. MMWR Morb Mortal Wkly Rep 53(17): 362-365.

40. Vujkovic M, Ocke MC, van der Spek PJ, Yazdanpanah N, Steegers EA, et al. (2007) Maternal Western dietary patterns and the risk of developing a cleft lip with or without a cleft palate. Obstet Gynecol 110(2 Pt 1): 378-384.

41. Piccoli GB, Attini R, Vasario E, Conijn A, Biolcati M, et al. (2010) Pregnancy and chronic kidney disease: a challenge in all CKD stages. Clin J Am Soc Nephrol 5(5): 844-855.

42. Cabiddu G, Castellino S, Gernone G, Santoro D, Moroni G, et al. (2016) A best practice position statement on pregnancy in chronic kidney disease: the Italian Study Group on Kidney and Pregnancy. J Nephrol 29(3): 277-303.

43. Piccoli GB, Fassio F, Attini R, Parisi S, Biolcati M, et al. (2012) Pregnancy in CKD: whom should we follow and why? Nephrol Dial Transplant 27(Suppl 3): iii111-iii118.

44. Vellanki K (2013) Pregnancy in chronic kidney disease. Adv Chronic Kidney 20(3): 223-228.

45. Gluba-Brzózka A, Franczyk B, Rysz J (2017) Vegetarian Diet in Chronic Kidney Disease-A Friend or Foe. Nutrients 9(4): pii: E374.

46. Piccoli GB, Attini R, Vasario E, Gaglioti P, Piccoli E, et al. (2011) Vegetarian supplemented low-protein diets. A safe option for pregnant CKD patients: report of 12 pregnancies in 11 patients. Nephrol Dia Transplant 26(1): 196-205.

47. Brenner B, Lawler E, Mackenzie H (1996) The hyperfiltration theory: a paradigm shift in nephrology. Kidney Int 49(6): 1774-1777.

48. Piccoli GB, Leone F, Attini R, Parisi S, Fassio F, et al. (2014) Association of low-protein supplemented diets with fetal growth in pregnant women with CKD. Clin J Am Soc Nephrol 9(5): 864-873.

49. Zaldivar MC, Peixoto A (2003) CKD series: cardiovascular risk reduction in patients with chronic kidney disease. Hosp Physician 39: 29-35.

50. Attini R, Leone F, Montersino B, Fassio F, Minelli F, et al. (2017) Pregnancy, Proteinuria, Plant-Based Supplemented Diets and Focal Segmental Glomerulosclerosis: A Report on Three Cases and Critical Appraisal of the Literature. Nutrients 9(7): pii: E770.

51. Attini R, Leone F, Parisi S, Fassio F, Capizzi I, et al. (2016) Veganvegetarian low-protein supplemented diets in pregnant CKD patients: fifteen years of experience. BMC Nephrol 17(1): 132.

52. Kniskern MA, Johnston CS (2011) Protein dietary reference intakes may be inadequate for vegetarians if low amounts of animal protein are consumed. Nutrition 27(6): 727-730.

53. Baroni L, Goggi S, Battaglino R Berveglieri M, Fasan I, et al. (2019) Vegan Nutrition for Mothers and Children: Practical Tools for Healthcare Providers. Nutrients 11(1): 5 .

54. Baroni L (2015) Vegetarianism in Food-Based Dietary Guidelines. Int J Nutr 1(2): 49-74.

55. Molloy A, Kirke P, Troendle J, Burke H, Sutton M, et al. (2009) Maternal vitamin B12 status and risk of neural tube defects in a population with high neural tube defect prevalence and no folic acid fortification Pediatrics 123(3): 917-23.
56. Balcı YI, Ergin A, Karabulut A, Polat A, Doğan M, et al. (2014) Serum vitamin B12 and folate concentrations and the effect of the Mediterranean diet on vulnerable populations. Pediatr Hematol Oncol 31(1): 62-67.

57. Pawlak R, Vos P, Shahab-Ferdows S, Hampel D, Allen LH, et al. (2018) Vitamin B-12 content in breast milk of vegan, vegetarian, and nonvegetarian lactating women in the United States. Am J Clin Nutr 108(3): 525-531.

58. Institute of Medicine Food Nutrition Board (2006) Dietary Reference Intakes: The Essential Guide to Nutrient Requirements. The National Academies Press, Washington DC, USA.

59. Institute of Medicine (US) (1990) Committee on Nutritional Status During Pregnancy and Lactation. Nutrition During Pregnancy: Part I Weight Gain: Part II Nutrient Supplements. National Academies Press, Washington DC, USA.

60. Branum AM, Bailey R, Singer BJ (2013) Dietary supplement use and folate status during pregnancy in the United States. J Nutr 143(4): 486492.

61. Picciano MF (2003) Pregnancy and lactation: physiological adjustments, nutritional requirements and the role of dietary supplements. J Nutr 133(6): 1997S-2002S.

62. Marra MV, Bailey RL (2018) Position of the Academy of Nutrition and Dietetics: Micronutrient Supplementation. J Acad Nutr Diet 118(11): 2162-2173.

63. Kominiarek MA, Rajan P (2016) Nutrition recommendations in pregnancy and lactation. Med Clin North Am 100(6): 1199-1215.

64. Rader JI, Yetley EA (2002) Nationwide folate fortification has complex ramifications and requires careful monitoring over time. Arch Intern Med 162(5): 608-609.

65. Yetley EA, Rader JI (2004) Modeling the level of fortification and post-fortification assessments: U.S. experience. Nutr Rev 62(6 Pt 2): S50-S59.

66. Gupta PM, Gahche JJ, Herrick KA, Ershow AG, Potischman N, et al. (2018) Use of Iodine-Containing Dietary Supplements Remains Low among Women of Reproductive Age in the United States: NHANES 2011-2014. Nutrients 10(4): E422.

67. Bailey RL, Pac SG, Fulgoni VL, Reidy KC, Catalano PM, et al. (2019) Estimation of Total Usual Dietary Intakes of Pregnant Women in the United States. JAMA Netw Open 2(6): e195967.

68. Pense J, Neher J, Kelsberg G (2015) Micronutrient Supplementation During Pregnancy. Am Fam Physician 92(3): 222-223.

69. Craig W, Mangels A, American Dietetic Association (2009) Position of the American Dietetic Association: vegetarian diets. J Am Diet Assoc 109(7): 1266-1282.

70. Sebastiani G, Barbero AH, Borrás-Novell C, Alsina Casanova M, AldecoaBilbao V, et al. (2019) The Effects of Vegetarian and Vegan Diet during Pregnancy on the Health of Mothers and Offspring. Nutrients 11(3): E557.

71. Rose S, Strombom A (2019) Ensuring adequate Vitamin B12 status on a plant-based diet. Adv Res Gastroentero Hapatol 13(3): 555862

72. Weaver CM, Proulx WR, Heaney R (1999) Choices for achieving adequate dietary calcium with a vegetarian diet. Am J Clin Nutr $70(3$ Suppl): 543S-548S

73. Sanders AB, Reddy S (1992) The influence of a vegetarian diet on the fatty acid composition of human milk and the essential fatty acid status of the infant. J Pediatr 120(4 Pt 2): S71-S77.

74. Messina V, Melina V, Mangels AR (2003) A new food guide for North American vegetarians. J Am Diet Assoc 103(6): 771-775. 
75. American Dietetic Association; Dietitians of Canada (2003) Position of the American Dietetic Association and Dietitians of Canada: Vegetarian diets. J Am Diet Assoc 103(6): 748-765

76. Slattery ML, Jacobs DR, Hilner JE, Caan B, Van Horn L, et al. (1991) Meat consumption and its associations with other diet and health factors in young adults: the CARDIA study. Am J Clin Nutr 54(5): 930-935.
77. Tesar R, Notelovitz M, Shim E, Kauwell G, Brown J, et al. (1992) Axial and peripheral bone density and nutrient intakes of postmenopausal vegetarian and omniv-orous women. Am J Clin Nutr 56(4): 699-704.

\section{Your next submission with Juniper Publishers will reach you the below assets}

- Quality Editorial service

- Swift Peer Review

- Reprints availability

- E-prints Service

- Manuscript Podcast for convenient understanding

- Global attainment for your research

- Manuscript accessibility in different formats

( Pdf, E-pub, Full Tsext, Audio)

- Unceasing customer service

Track the below URL for one-step submission https://juniperpublishers.com/online-submission.php 